PROCEEDINGS OF THE

AMERICAN MATHEMATICAL SOCIETY

Volume 125, Number 12, December 1997, Pages 3715-3720

S 0002-9939(97)04088-4

\title{
CO-ELEMENTARY EQUIVALENCE, CO-ELEMENTARY MAPS, AND GENERALIZED ARCS
}

\author{
PAUL BANKSTON
}

(Communicated by Andreas R. Blass)

\begin{abstract}
By a generalized arc we mean a continuum with exactly two non-separating points; an arc is a metrizable generalized arc. It is well known that any two arcs are homeomorphic (to the real closed unit interval); we show that any two generalized arcs are co-elementarily equivalent, and that co-elementary images of generalized arcs are generalized arcs. We also show that if $f: X \rightarrow Y$ is a function between compacta and if $X$ is an arc, then $f$ is a co-elementary map if and only if $Y$ is an arc and $f$ is a monotone continuous surjection.
\end{abstract}

\section{INTRODUCTION AND OUTLINE OF RESUlTS}

All topological spaces under consideration are Hausdorff; a compactum is a Hausdorff space that is compact. A generalized arc is a continuum (i.e., a connected compactum) that has exactly two non-separating points; an arc is a metrizable generalized arc. The class of generalized arcs is precisely the class of linearly orderable continua, each generalized arc admitting exactly two compatible linear orders. The class of (continuous images of) generalized arcs has been extensively studied over the years (see [10], [11], [14]), the most well-known results in this area being that any two arcs are homeomorphic (to the standard closed unit interval on the real line), and (Hahn-Mazurkiewicz) that a Hausdorff space is a continuous image of an arc if and only if that space is a locally connected metrizable continuum. In this paper, a continuation of [3], we study the model-theoretic topology of generalized arcs, in particular, the "dualized model theory" of these spaces.

Many notions from classical first-order model theory, principally elementary equivalence and elementary embedding, may be phrased in terms of mapping conditions involving the ultraproduct construction. Because of the (Keisler-Shelah) ultrapower theorem (see, e.g., [7]), two relational structures are elementarily equivalent if and only if some ultrapower of one is isomorphic to some ultrapower of the other; a function from one relational structure to another is an elementary embedding if and only if there is an ultrapower isomorphism so that the obvious square mapping diagram commutes (see, e.g., [2], [5], [8]). The ultrapower construction in turn is a direct limit of direct products, and is hence capable of being transferred

Received by the editors July 2, 1996.

1991 Mathematics Subject Classification. Primary 03C20, 54B35, 54C10, 54D05, 54D30, 54D $80,54 \mathrm{~F} 05,54 \mathrm{~F} 15$.

Key words and phrases. Ultraproduct, ultracoproduct, generalized arc, co-elementary equivalence, co-elementary map.

(C)1997 American Mathematical Society 
into a purely category-theoretic setting. In this paper we focus on the category CH of compacta and continuous maps, but perform the transfer into the opposite category (thus justifying the phrase "dualized model theory" above).

In $\mathbf{C H}$ one then constructs ultracoproducts, and talks of co-elementary equivalence and co-elementary maps. Co-elementary equivalence is known [2], [5], [9] to preserve important properties of topological spaces, such as being infinite, being connected, being Boolean (i.e., totally disconnected), having (Lebesgue) covering dimension $n$, and being a decomposable continuum. If $f: X \rightarrow Y$ is a co-elementary map in $\mathbf{C H}$, then of course $X$ and $Y$ are co-elementarily equivalent (in symbols $X \equiv Y$ ). Moreover, since $f$ is a continuous surjection (see [2]), additional information about $X$ is transferred to $Y$. For instance, continuous surjections in $\mathbf{C H}$ cannot raise weight (i.e., the smallest cardinality of a possible topological base, and for many reasons the right cardinal invariant to replace cardinality in the dualized model-theoretic setting), so metrizability (i.e., being of countable weight in the compact Hausdorff context) is preserved. Also local connectedness is preserved, since continuous surjections in $\mathbf{C H}$ are quotient maps. Neither of these properties is an invariant of co-elementary equivalence alone.

When attention is restricted to the full subcategory of Boolean spaces, the dualized model theory matches perfectly with the model theory of Boolean algebras because of Stone duality. In the larger category there is no such match [1], [12], however, and one is forced to look for other (less direct) model-theoretic aids. Fortunately there is a finitely axiomatizable Horn class of bounded lattices, the so-called normal disjunctive lattices [6] (also called Wallman lattices in [5]), comprising precisely the (isomorphic copies of) lattices that serve as bases for the closed sets of compacta. We go from lattices to spaces, as in the case of Stone duality, via the maximal spectrum $S($ ), pioneered by H. Wallman [13]. $S(A)$ is the space of maximal proper filters of $A$; a typical basic closed set in $S(A)$ is the set of elements of $S(A)$ containing a given element of $A$. $S()$ is contravariantly functorial; if $f: A \rightarrow B$ is a homomorphism of normal disjunctive lattices and $M \in S(B)$, then $f^{S}(M)$ is the unique maximal filter in $A$ containing the pre-image of $M$ under $f$. It is a fairly straightforward task to show, then, that $S()$ converts ultraproducts to ultracoproducts, elementarily equivalent lattices to co-elementarily equivalent compacta, and elementary embeddings to co-elementary maps (see [2], [4], [5], [6], [9]). An important consequence of this is a Löwenheim-Skolem theorem for co-elementary maps: every compactum maps co-elementarily onto a metrizable compactum. (This result is used in 2.4 and 2.6 below.)

In [3] we showed that any locally connected metrizable space co-elementarily equivalent to an arc is already an arc; here we present the following results. (i) if $f$ : $X \rightarrow Y$ is a co-elementary map in $\mathbf{C H}$, and if $Y$ is locally connected (in particular, a generalized arc), then $f$ is a monotone continuous surjection; (ii) co-elementary images of (generalized) arcs are (generalized) arcs; (iii) any two generalized arcs are co-elementarily equivalent; (iv) if $X$ is a generalized arc and $f: X \rightarrow Y$ is an irreducible co-elementary map in $\mathbf{C H}$, then $f$ is a homeomorphism; (v) if every locally connected co-elementary pre-image of an arc is a generalized arc, then every locally connected compactum co-elementarily equivalent to a generalized arc is also a generalized arc; and (vi) if $X$ is an arc and $f$ is a function from $X$ to a compactum $Y$, then $f$ is a co-elementary map if and only if $Y$ is an arc and $f$ is a monotone continuous surjection. 
Local connectedness is necessarily a part of (v) above. We do not know at present whether the hypothesis in (v) is true, nor do we know whether monotone surjections between generalized arcs are always co-elementary maps.

Remark 1.1. By way of contrast, there is a Boolean analogue to some of the results above. Define a generalized Cantor set to be any non-empty Boolean space with no isolated points, and a Cantor set to be a metrizable generalized Cantor set. It is well known that any two Cantor sets are homeomorphic (to the standard Cantor middle thirds set in the real line), and that the generalized Cantor sets are precisely the Stone duals of the atomless Boolean algebras, constituting an elementary class whose first-order theory is $\aleph_{0}$-categorical, complete, and model complete. In (ii) and (iii), one may replace "arc" with "Cantor set" uniformly; a straightforward application of $\aleph_{0}$-categoricity. The analog of (iv) is false (see Example 3.3.4(iv) in [2]); the projective cover map to a generalized Cantor set is always an irreducible co-elementary map between (seldom-homeomorphic) generalized Cantor sets. As for (v), it follows from the results on dimension in [2] that any compactum coelementarily equivalent to a generalized Cantor set is itself a generalized Cantor set. Finally, regarding (vi), all continuous surjections between generalized Cantor sets are co-elementary maps. This is a direct consequence of the model completeness of the theory of atomless Boolean algebras.

\section{Methods AND PROOFS}

We begin with a proof of (i) above. Recall that a map $f: X \rightarrow Y$ is monotone if the inverse image of a point of $Y$ is connected in $X$. It is easy to check that inverse images of closed connected sets under monotone quotient maps are connected. (If $A_{1} \cup A_{2}$ is a disconnection of the inverse image $f^{-1}[K]$, then both $A_{1}$ and $A_{2}$ are closed in $X$; also they are $f$-saturated because of monotonicity. Thus $A_{i}=$ $f^{-1}\left[f\left[A_{i}\right]\right], i=1,2$. Because $f$ is a quotient map, $f\left[A_{1}\right] \cup f\left[A_{2}\right]$ is a disconnection of $K$.) Since continuous maps between compacta are closed, monotone continuous surjections pull subcontinua back to subcontinua.

Proposition 2.1. Let $f: X \rightarrow Y$ be a co-elementary map in $\boldsymbol{C H}$, with $Y$ locally connected. Then $f$ is a monotone continuous surjection.

Proof. Assume $f: X \rightarrow Y$ is co-elementary, $Y$ is locally connected, and $f$ is not monotone. Then there is a point $y \in Y$ such that the fiber $A:=f^{-1}[\{y\}]$ is disconnected, say, into two disjoint nonempty closed sets $A_{1}$ and $A_{2}$. For $i=1,2$, let $U_{i}$ be an open neighborhood of $A_{i}$, with $U_{1} \cap U_{2}=\emptyset$. If $C$ is a subcontinuum of $X$ containing $A$, then we can pick some $x_{C} \in C \backslash\left(U_{1} \cup U_{2}\right)$. Let $B$ be the closure of the set of all such points $x_{C}$, as $C$ ranges over all subcontinua containing $A$. Since no point $x_{C}$ lies in $U_{1} \cup U_{2}, B$ is disjoint from $A$, but intersects every subcontinuum of $X$ that contains $A$.

Now $f[B]$ is closed in $Y$ and does not contain $y$. Let $V$ be an open neighborhood of $y$ whose closure $K$ misses $f[B]$. Because $Y$ is locally connected, we may take $V$ to be connected; hence $K$ is a subcontinuum of $Y$ containing $y$ in its interior. We need a fact proved elsewhere.

Lemma (Lemma 2.8 in [5]). Let $f: X \rightarrow Y$ be a co-elementary map in $\boldsymbol{C H}$, with $K \subseteq Y$ a subcontinuum. Then there is a subcontinuum $C \subseteq X$ such that $K=f[C]$, and whenever $V \subseteq K$ is open in $Y, f^{-1}[V] \subseteq C$. 
Using the lemma, there exists a subcontinuum $C \subseteq X$ such that $f[C]=K$ and $f^{-1}[V] \subseteq C$. Let $x \in A$. Then there is a neighborhood $U$ of $x$ with $f[U] \subseteq V$. Thus $x \in U \subseteq f^{-1}[V] \subseteq C$, hence we infer $A \subseteq C$. Every subcontinuum of $X$ containing $A$ must intersect $B$, so $\emptyset \neq f[B \cap C] \subseteq f[B] \cap f[C]=f[B] \cap K=\emptyset$. This contradiction completes the proof.

Remark 2.2. The lemma above provides only a weak consequence of co-elementarity. Indeed, the usual projection map from the standard closed unit square in the plane onto its first coördinate is not co-elementary because it does not preserve topological dimension. Nevertheless, it does satisfy the conclusion of the lemma.

Now we are in a position to prove (ii).

Proposition 2.3. Let $f: X \rightarrow Y$ be a co-elementary map in $\boldsymbol{C H}$. If $X$ is a generalized arc, then so is $Y$.

Proof. Let $f: X \rightarrow Y$ be a co-elementary map in $\mathbf{C H}$, with $X$ a generalized arc. $Y$ is a locally connected continuum because $X$ is locally connected and $f$ is a continuous surjection. By 2.1, $f$ is monotone; it remains to show $Y$ has precisely two non-separating points.

Let $a, b \in X$ be the two non-separating points of $X$. $Y$ is non-degenerate because of co-elementarity; monotonicity then tells us that $f(a) \neq f(b)$. If $f(a)$ were to separate $Y$, we could also separate $X \backslash K$, where $K:=f^{-1}[\{f(a)\}]$ is a subcontinuum (i.e., closed subinterval) containing the endpoint $a$. This is easily seen to be impossible for generalized arcs. Now let $y \in Y \backslash\{f(a), f(b)\}$, with $K:=f^{-1}[\{f(y)\}]$. Then $K$ is a subcontinuum of $X$ containing neither endpoint. Thus $X \backslash K$ is disconnected; hence $y$ separates $Y$. We therefore conclude that $Y$ is a generalized arc.

We can very quickly settle (iii).

Proposition 2.4. Let $X$ and $Y$ be two generalized arcs. Then $X \equiv Y$.

Proof. Let $X$ and $Y$ be generalized arcs. By the Löwenheim-Skolem theorem for co-elementary maps, there exist co-elementary maps $f: X \rightarrow X_{0}$ and $g: Y \rightarrow Y_{0}$, where $X_{0}$ and $Y_{0}$ are compact metrizable. By 2.3, the images are generalized arcs; hence they are arcs. Thus $X_{0}$ and $Y_{0}$ are homeomorphic, and we conclude $X \equiv Y$ because [2] co-elementary equivalence is an honest equivalence relation.

To handle (iv), recall that a continuous surjection $f: X \rightarrow Y$ is irreducible if $Y$ is not the image under $f$ of a proper closed subset of $X$.

Proposition 2.5. Let $f: X \rightarrow Y$ be an irreducible co-elementary map in $\boldsymbol{C H}$. If $X$ is a generalized arc, then $f$ is a homeomorphism.

Proof. It suffices to show $f$ is one-one. Let $y \in Y$, with $K:=f^{-1}[\{y\}]$, a subcontinuum of $X$ by 2.1. Since $X$ is a generalized arc, $K$ is either a singleton or a closed subinterval with non-empty interior. The latter case easily contradicts the irreducibility of $f$, however.

In [9] it is shown that every infinite compactum is co-elementarily equivalent to a compactum that is not locally connected. (See also [5] for refinements.) This explains the necessity of the local connectedness hypothesis in (v). 
Proposition 2.6. Suppose every locally connected co-elementary pre-image of an arc is a generalized arc. Then every locally connected compactum co-elementarily equivalent to a generalized arc is itself a generalized arc.

Proof. Suppose $X \in \mathbf{C H}$ is locally connected, $X \equiv Y$, and $Y$ is a generalized arc. As in the proof of 2.4 above, we have co-elementary maps $f: X \rightarrow X_{0}$ and $g: Y \rightarrow Y_{0}$, where $X_{0}$ and $Y_{0}$ are metrizable. Furthermore, we know that $X_{0}$ is locally connected and that $Y_{0}$ is an arc (2.1 again). By the transitivity of coelementary equivalence, we know $X_{0} \equiv Y_{0}$; by the main result of [3], we know $X_{0}$ is an arc. Our hypothesis then tells us that $X$ is a generalized arc.

We finish with a proof of (vi). If $X$ is an arc and $f: X \rightarrow Y$ is a co-elementary map in $\mathbf{C H}$, then $Y$ is an arc and $f$ is a monotone continuous surjection by 2.1 and 2.2. So it suffices to prove the following.

Proposition 2.7. Every monotone continuous surjection from an arc to itself is a co-elementary map.

Proof. Let us take our arc to be the standard closed unit interval I with its usual order. $f$ is either $\leq$-preserving or $\leq$-reversing, so we lose no generality in assuming $f$ to be the former.

For any topological space $X$, we denote the closed set lattice of $X$ by $F(X)$. $F($ ) converts continuous maps contravariantly into lattice homomorphisms, and serves as a right inverse for $S(): S(F(X))$ is naturally homeomorphic to $X$ for any compactum $X$.

Since $f$ is a monotone continuous surjection, $f^{F}: F(\mathbf{I}) \rightarrow F(\mathbf{I})$ is a lattice embedding that takes closed intervals (in this case the connected elements of the lattice) to closed intervals. However, $f^{F}$ will take atoms to non-atoms when $f$ is not injective. Thus $f^{F}$ is not an elementary embedding without being an isomorphism. The idea is to restrict the domain and range of $f^{F}$ in such a way that the resulting lattice embedding, call it $g$, is elementary, and $g^{S}=f$.

Our plan is to create an elementary lattice embedding $g: \mathcal{A} \rightarrow \mathcal{B}$, where $\mathcal{A}$ and $\mathcal{B}$ are atomless lattice bases for $\mathbf{I}$ (i.e., both $\mathcal{A}$ and $\mathcal{B}$ are atomless, as well as meet-dense in $F(\mathbf{I})$ ), and $g$ agrees with the restriction of $f^{F}$ to $\mathcal{A}$.

Since $S(\mathcal{A})$ and $S(\mathcal{B})$ are naturally homeomorphic to $\mathbf{I}$, and $f$ is just $g^{S}$ conjugated with these homeomorphisms, $f$ is a co-elementary map provided $g^{S}$ is.

For each $y \in \mathbf{I}$, let $\lambda(y):=\inf \left(f^{-1}[\{y\}]\right)$ and $\rho(y):=\sup \left(f^{-1}[\{y\}]\right)$. Then for any closed interval $[x, y] \in F(\mathbf{I}), f^{F}([x, y])=[\lambda(x), \rho(y)]$. Both $\lambda$ and $\rho$ are right inverses for $f$, and are hence strictly increasing (but not necessarily continuous). Of course $\lambda(0)=0$ and $\rho(1)=1$.

Let $L, R \subseteq \mathbf{I}$, with $0 \in L$ and $1 \in R$. If $\mathcal{I}(L, R)$ denotes the set of all finite unions of intervals $[x, y]$ with $x \in L$ and $y \in R$, then $\mathcal{I}(L, R)$ is a sublattice of $F(\mathbf{I})$, which is atomless just in case $L \cap R=\emptyset$. If $L$ and $R$ are dense in $\mathbf{I}$, then $\mathcal{I}(L, R)$ is a lattice base as well.

Now fix $L, R \subseteq \mathbf{I}$ to be disjoint countable dense subsets, with $0 \in L$ and $1 \in R$, and set $\mathcal{A}:=\mathcal{I}(L, R)$. Then the image of $\mathcal{A}$ under $f^{F}$ is $\mathcal{I}(\lambda[L], \rho[R])$. Clearly $\lambda[L] \cap \rho[R]=\emptyset, 0 \in \lambda[L]$, and $1 \in \rho[R]$. Let $L^{\prime}, R^{\prime} \subseteq \mathbf{I}$ be disjoint countable dense subsets, with $\lambda[L] \subseteq L^{\prime}, \rho[R] \subseteq R^{\prime}$, and set $\mathcal{B}:=\mathcal{I}\left(L^{\prime}, R^{\prime}\right)$. Then $\mathcal{B}$ is a countable atomless lattice base for $F(\mathbf{I})$, and we denote by $g: \mathcal{A} \rightarrow \mathcal{B}$ the embedding $f^{F}$ with its domain and range so restricted. It remains to show that $g$ is an elementary embedding, and for this it suffices to show that for each finite set $S$ in $\mathcal{A}$ and each 
$b \in \mathcal{B}$, there is an automorphism on $\mathcal{B}$ that fixes $g[S]$ pointwise and takes $b$ into $g[\mathcal{A}]$.

Let $x_{1}, \ldots, x_{n}$ be a listing, in increasing order, of the endpoints of the component intervals of $g[S] \cup\{b\}$ ( so each $x_{i}$ is in $L^{\prime} \cup R^{\prime}$ ), with $X_{i}:=f^{-1}\left[\left\{f\left(x_{i}\right)\right\}\right], 1 \leq i \leq n$. Each $X_{i}$ is either a singleton or a non-degenerate closed interval, and for $1 \leq i<$ $j \leq n$, either $X_{i}=X_{j}$ or each element of $X_{i}$ is less than each element of $X_{j}$. Let $U_{i}$ be an open-interval neighborhood of $X_{i}$ such that $U_{i} \cap U_{j}=\emptyset$ whenever $X_{i} \neq X_{j}$. Since $f$ is a $\leq$-preserving surjection and the sets $L$ and $R$ are dense in $\mathbf{I}$, each $U_{i}$ has infinite intersection with both $\lambda[L]$ and $\rho[R]$. If $x_{i} \in \lambda[L] \cup \rho[R]$, set $x_{i}^{\prime}:=x_{i}$. Otherwise we know $x_{i}$ is an endpoint of a component interval of $b$; and we choose $x_{i}^{\prime} \in U_{i}$ in such a way that $x_{i}^{\prime} \in \lambda[L]$ if and only if $x_{i} \in L^{\prime}$, and $x_{i}^{\prime}<x_{j}^{\prime}$ whenever $x_{i}<x_{j}$ and $X_{i}=X_{j}$. This procedure produces an increasing sequence $x_{1}^{\prime}, \ldots, x_{n}^{\prime}$ of elements of $\lambda[L] \cup \rho[R] ; x_{i}^{\prime} \in \lambda[L]$ if and only if $x_{i} \in L^{\prime}$. For each $a \in g[S] \cup\{b\}$, let $a^{\prime}$ be built up using the endpoints $x_{i}^{\prime}$ in the same way as $a$ is built up using the endpoints $x_{i}$. Then $a^{\prime}=a$ for each $a \in g[S]$, and $b^{\prime} \in g[\mathcal{A}]$. Now by a classic (Cantor) back and forth argument, there is an order automorphism on $L^{\prime} \cup R^{\prime}$ that fixes $L^{\prime}$ and $R^{\prime}$ setwise and takes $x_{i}$ to $x_{i}^{\prime}$ for $1 \leq i \leq n$. This order automorphism gives rise to the lattice automorphism on $\mathcal{B}$ that we require.

\section{REFERENCES}

1. B. Banaschewski, "More on compact Hausdorff spaces and finitary duality," Can. J. Math. 36(1984), 1113-1118. MR 86h: 18002

2. P. Bankston, "Reduced coproducts of compact Hausdorff spaces," J. Symbolic Logic 52(1987), 404-424. MR 88k:54021

3. — "Model-theoretic characterizations of arcs and simple closed curves," Proc. A. M. S. 104(1988), 898-904. MR 89m:03023

4. - "Co-elementary equivalence for compact Hausdorff spaces and compact abelian groups," J. Pure and Applied Algebra 68 (1990), 11-26. MR 91k:03095

5. — "Taxonomies of model-theoretically defined topological properties," J. Symbolic Logic 55(1990), 589-603. MR 91g:03065

6. — , "On the topological model theory of normal disjunctive lattices," (submitted)

7. C. C. Chang and H. J. Keisler, "Model Theory (Third Edition)," North Holland, Amsterdam, 1989. MR 91c:03026

8. P. Eklof, "Ultraproducts for algebraists," in "Handbook of Mathematical Logic," North Holland, Amsterdam, 1977, pp. 105-137.

9. R. Gurevič, "On ultracoproducts of compact Hausdorff spaces," J. Symbolic Logic 53(1988), 294-300. MR 89b:03051

10. J. G. Hocking and G. S. Young, "Topology," Addison-Wesley, Reading, MA, 1961. MR 23:A2857

11. J. Nikiel, H. M. Tuncali, and E. D. Tymchatyn, "Continuous images of arcs and inverse limit methods," Memoirs of the A. M. S. 104 (no. 498) (1993), 1-80. MR 94a:54084

12. J. Rosický, "Categories of models," Seminarberichte Mathematik Informatik Fernuniversität 19(1984), 337-413.

13. H. Wallman, "Lattices and topological spaces," Ann. Math.(2) 39(1938), 112-126.

14. S. Willard, "General Topology," Addison-Wesley, Reading, MA, 1970. MR 41:9173

Department of Mathematics, Statistics and Computer Science, Marquette UniverSity, Milwaukee, Wisconsin 53201-1881

E-mail address: paulb@mscs.mu.edu 